\title{
A Medical Simulator for Subcutaneous Contraceptive Implant Insertion
}

\author{
A. Jardin, M.T. Pham, A. Mallet, T. Redarce \\ Laboratoire AMPERE, UMR CNRS 5005, INSA-Lyon, F-69621, France
}

O. Dupuis

Centre Hospitalier Universitaire Lyon Sud, Pierre-Bénite, F-69495, France

\begin{abstract}
New contraceptive methods like the subcutaneous implant offers a new kind of comfort for women with an efficiency similar to the contraceptive pill. Unfortunately the few numbers of unintended pregnancies that have been reported are generally due to a bad insertion of the implant. In order to give more security to patients, we have designed, in close collaboration with physicians, a new kind of medical simulator. This paper focuses on a device dedicated to a specific subcutaneous implant but it is worth noting that this simulator is relatively generic since it will be used for other subcutaneous techniques or other implant instruments. This simulator can be used for two purposes: one for training novice physicians in the correct manipulation and the other for doctor certification which will help determine if they are capable of inserting the implant in vivo. This paper describes the approach which has led to the design of this simulator. It describes its functionalities, its several components but also methods used to analyze the manipulation of the implant insertion inside the patient. Finally first experimental results are reported and discussed. The system used in this paper makes possible to carry out training in a context free of constraints and provides the first mean of visualizing a maneuver that, until now, has been performed blindly.
\end{abstract}

Key words: Medical simulator, Contraceptive implant, Training tool, Path analysis, Gynecology

Email addresses: $\{$ audrey.jardin, minh-tu.pham\}@insa-lyon.fr (A. Jardin, M.T. Pham, A. Mallet, T. Redarce), olivier.dupuis@chu-lyon.fr (O. Dupuis). 


\section{Introduction}

With significant progress in new technologies and modern techniques in miniinvasive surgery, the introduction of medical simulators is gaining an increasing appeal in the clinical environment. These tools allow the visualization of the position of organs, the planning of surgical interventions, and the carrying out of more comprehensive postoperative monitoring. The flexibility of such tools also permits to reset some parameters in order to adapt the medical intervention to each patient's data. In fact medical simulators take into account the needs of physicians but also the ethical problems and risks that patients may be exposed to during the learning period of a new technique. The main interest of these tools is to train inexperienced physicians without putting the patients' well-being in danger. This can be especially useful in gynecology and more particularly for contraceptive implant insertion. Indeed, despite a comfort and an efficiency similar to classic contraceptive methods, in the case of the subcutaneous implant, some cases of pregnancy have been reported. A survey concerning these contraception failures [1] shows that "in France 39 unintended pregnancies were reported over 17 months" and were "in $77 \%$ of cases due to an insertion technique error". This underlines the lack and the necessity of such a training tool. In this context, we have developed, in collaboration with physicians, a new medical simulator that offers new solutions for doctors' training. This simulator could allow young professionals to acquire experience in this specific manipulation. A simulator training can also be used to validate new instruments, new techniques and to evaluate capacities. One of the advantages of a simulator is to bypass the constraints occurring in an actual insertion. These constraints are:

- the manipulation occurs inside the arm and thus a novice does not see the instructor's manipulations and reciprocally instructors can not validate the novice's manipulations;

- the first implant insertions carried out by novices are always awkward because of stress linked to the risks of such an operation.

This paper highlights the design of an innovative medical simulator. A task analysis has been done in Section 2 and the advantages of an appropriate simulator used to reproduce and learn the task are shown. The specifications of the simulator have been analyzed and translated in terms of mechanical, hardware, and software specifications in Section 3. Experimental results are presented and discussed in Section 4. 


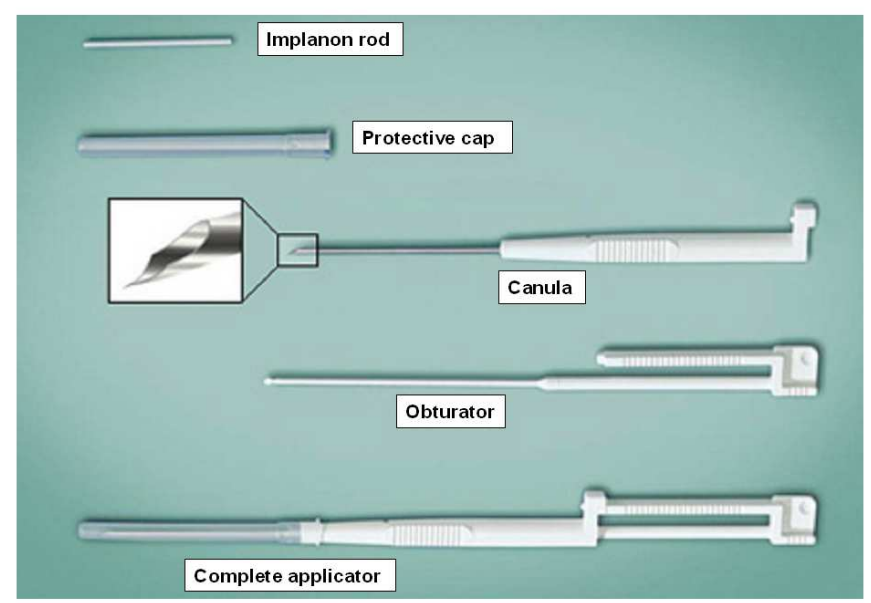

Fig. 1. The device used for implant insertion

\section{Task description}

This paper focuses on a device dedicated to a specific subcutaneous implant: Implanon. This implant works like the so-called minipill (especially the one called Cerazette) and provides contraceptive protection for up to 3 years when it is inserted subdermally [2-4]. Thus women do not worry anymore about forgetting their contraceptive method. The implant consists of a single-rod $40 \mathrm{~mm}$ long and $2 \mathrm{~mm}$ in diameter containing etonogestrel which is progressively diffused. After a local anaesthetic, the physician inserts it through a sterile applicator (Fig. 1) into the upper-arm, 60-80 $\mathrm{mm}$ above the elbow [5]. He introduces the needle directly under the skin, turns the obturator by $90^{\circ}$ and finally pulls out the canula while he keeps the obturator fixed. Even if the manipulation for the implant insertion seems to be very easy, there are several points the physician has to check [6]:

- the insertion has to be made within the first five days following the beginning of a menstrual bleeding;

- there must not have been medicinal interaction prior to the procedure;

- the implant has to be inserted in the good location inside the arm as recommended by Organon the manufacturer of Implanon.

In fact the correctness of the insertion determines the ease of the implant removal. If the implant is correctly inserted then it is palpable in the sulcus between the biceps and the triceps. On the contrary, if the implant is not well inserted, it remains impalpable and therefore difficult to find and remove. In this case, the bad placement can induce some pain for the patient, a lesser efficiency and above all, it can lead to a severe surgical intervention in order to remove the implant [7-9]. It is essential that the manipulation is accomplished in the skin layers at a limited depth: the implant rod must not be placed into 
the limit of the muscular tissue (called aponeurosis). If we quantify the limited depth to 3-4 mm (which corresponds approximately to the thickness of the skin for an average patient), the physician has a very small margin of error, compared to the $2.5 \mathrm{~mm}$-diameter of the canula. This accuracy is all the more difficult to obtain since:

- the thickness of the skin depends on the patient's morphology (and particularly concerning the adipose layer) (Fig. 2);

- up until now specific training to this kind of manipulation did not exist: during his first experience of implant insertion, the physician can only learn with the user's implant guide.

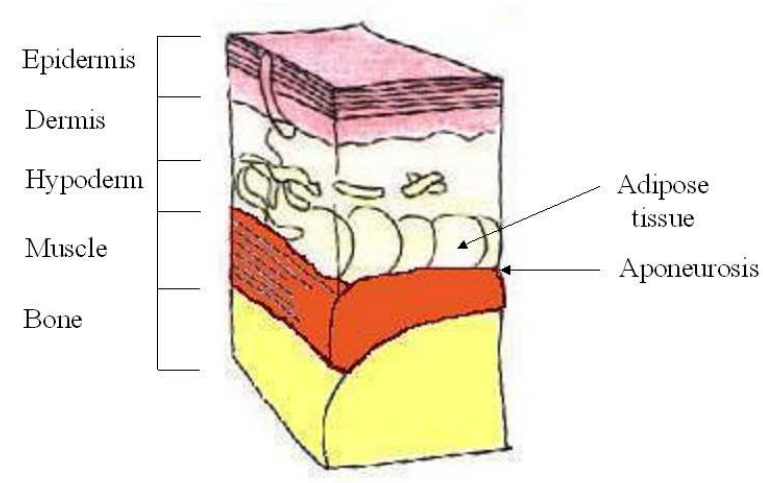

Fig. 2. Diagram of the different skin layers

Given the high interest in improving insertion conditions for contraceptive implants, the design of a simulator has acquired a high level of relevancy for the inexperienced physicians who wish to learn a better insertion technique [10].

\section{Materials and methods}

\subsection{Operating modes}

The simulator is mainly composed of two components: a mechanical and a software component. The material component consists of a dummy human arm, an insertion applicator and a computer equipped with a data acquisition system. This component ensures a physical support to the training and consti- 
tutes a haptic interface. The second component is a visual interface projected on a computer screen with which the student can see inside the arm in realtime: this option helps the novice to learn how to control his manipulation and to see the correct placement of the implant. Moreover, as we mentioned earlier, the simulator was designed both for learning the good manipulation but also for delivering an implant insertion certificate. So it is designed to several kinds of operators. The operator may be a physician teacher who explains the technique to his students as well as an inexperienced physician training him or herself in the insertion of the implant, or as a participant of a certification exam. Thus, with this objective, two operating modes are proposed, a training and a certification mode:

- The training mode allows an instructor to teach novices the correct manipulation. The student is then guided by a graphical interface which can simultaneously display the actual instrument path and the path to trail. In this mode, operator and simulator interact together at the same time.

- On the contrary, the certification mode allows the instructor to evaluate the physicians trained in the insertion technique. The participant of the certificate program carries out his manipulation on the mechanical model without any assistance or graphical interface. The actual operator trajectory is then recorded and compared to a reference manipulation. According to judiciously selected criteria, the examiner can decide if the candidate is capable of inserting a contraceptive implant on real patients. In this mode, the operator does not interact simultaneously with the software component of the simulator. The software component is used only for an a posteriori data treatment.

\subsection{Simulator components}

In order to obtain the best results, the main requirements have been firstly defined in close collaboration with a medical team. It has been underlined that:

- the material component of the simulator must be as realistic as possible;

- the operator must have the possibility to move the arm in order to choose the right position and the insertion site;

- the visual component must provide a graphical interface that allows the operator to watch the path of his instrument needle tip inside the arm;

- the system must be able to record operators' paths and calculate errors between these data and a reference path.

The different components of the simulator have then be designed in accordance with these needs. 


\subsubsection{Mechanical component}

The mechanical component consists of an anthropomorphic arm and its fixation system. The arm model was manufactured by the Nasco Corporation [11]. It accurately reproduces an actual arm with its particular anatomical landmarks. The base of the simulator is a wooden table on which the arm is attached. The fastening device of the arm is made of two parts: one at the shoulder level and another one at the wrist level. So as to obtain a model as stiff as possible and to ensure a good correlation between the actual arm position and the position of its numerical model, the shoulder is attached to a PVC pivot joint and an indexing system (Fig. 3). The pivot axis runs from the arm to facilitate its rotation while the indexing mechanism allows to block the arm rotation.

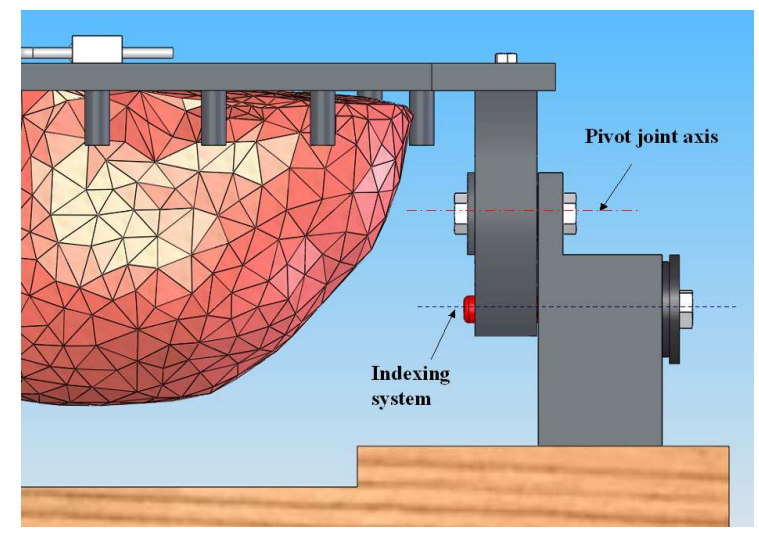

Fig. 3. Arm-support joint at the level of the shoulder

At the wrist, a PVC bracelet with five pins has been designed (Fig. 4). The pins allow the placement of the arm in five different orientations.

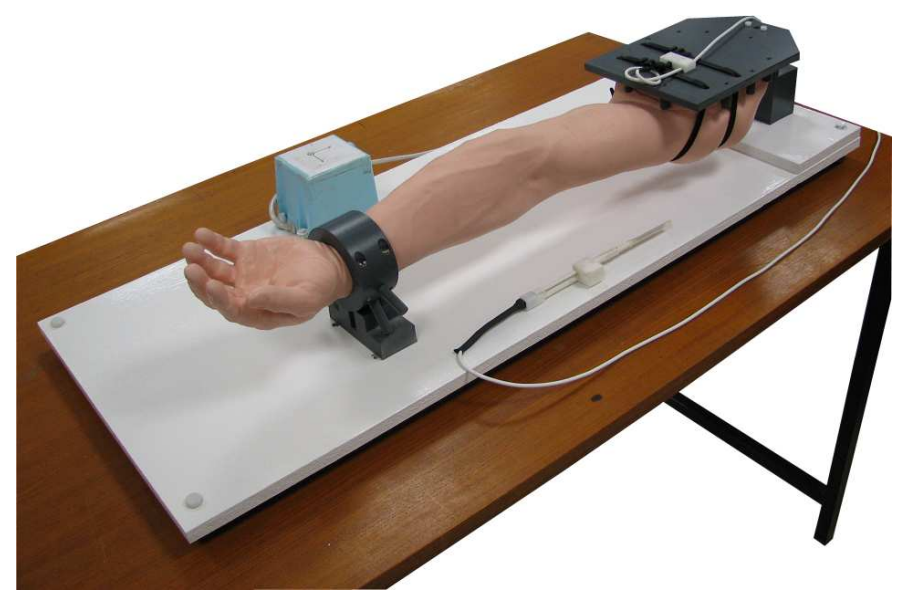

Fig. 4. The bracelet of the wrist 


\subsubsection{Miniaturized position sensors}

The originality of our simulator is that it makes it possible to study the needle tip paths inside the arm, allowing the medical team to have a better understanding of the manipulation of the subcutaneous implant placement. To monitor the simulator various components, several challenges had to be overcome: especially the restricted workspace and the obstruction of some objects which means they can not be monitored inside the arm. To solve this problem, we chose a system using six degrees of freedom electromagnetic sensors called the MiniBird system which is developed by Ascension company. This system can track the position and orientation of masked objects [12]. These sensors measure the impulse of the magnetic field emitted by a box called a transmitter. Three factors must be taken into account when using such a system: the presence of ferromagnetic materials in the measurement field can disrupt measurements; the measurement field is limited in size; and the 120 $\mathrm{Hz}$ sampling rate is divided by the number of sensors used. The first constraint is solved using non-magnetic materials for the simulator (wood, PVC, ABS). The second constraint can be solved using appropriate sensors for the task. Here the workspace dimension of the sensors (a 80-cm diameter half-sphere) is more than sufficient because data acquisition takes place inside or beside the arm. Finally the last constraint leads us to check the compatibility of the sensor dynamics with respect to the velocity of the manipulation. Since we are using three sensors (one in the shoulder of the arm and two in the instrument used for implant insertion), the sampling rate is $40 \mathrm{~Hz}$. This frequency is compatible with classic implant placement. Concerning the analysis of data, the Controldesk software provided with a D-Space data acquisition board allowed us to collect the flow of data stemming from the sensors $[13,14]$.

\subsubsection{Instrumented tool for implant insertion}

Since the positions, the orientation of the arm and also the two parts of the insertion tool (i.e. the obturator and applicator) have to be known during the entire time of the simulation, there is a need of two adaptation pieces for placing sensors (Fig. 5). Each of them was designed:

- in ABS by rapid prototyping in order to avoid magnetic disturbance on sensor measurement;

- with a small lodge for sensor location;

- removable but ensuring a repeating placement of the sensor with respect to the instrument;

- small so as to hamper the medical manipulation the least possible and to shun any collision when sliding the applicator along the obturator (Fig. 6). 


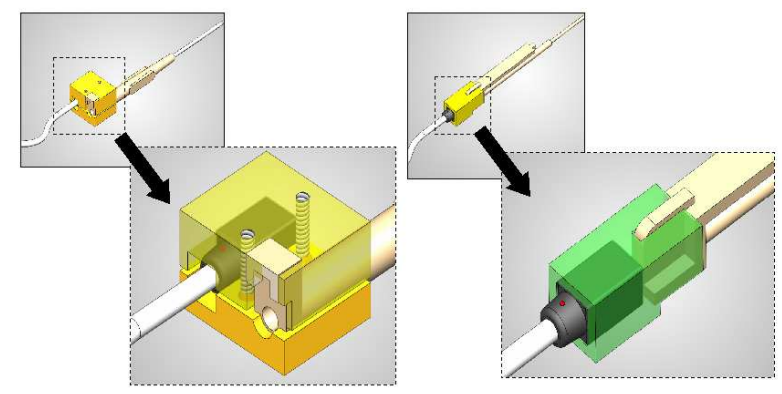

Fig. 5. Lodges for the 3D position sensors

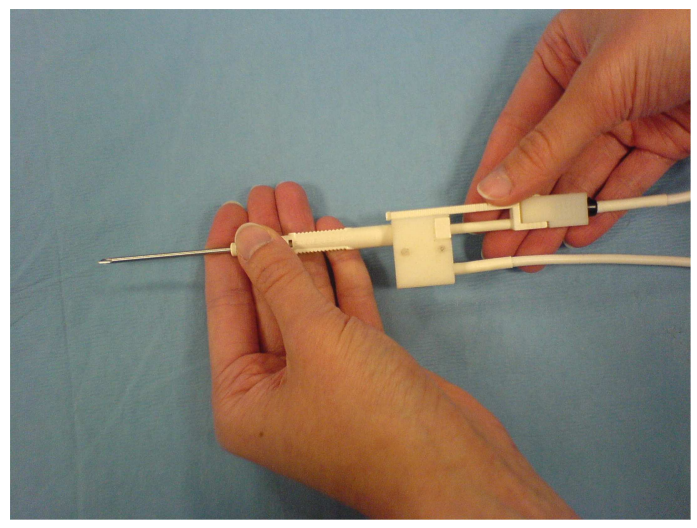

Fig. 6. Instrumented tool used for implant insertion

\subsubsection{Visual Component}

Nowadays simulators provided with a $3 \mathrm{D}$ visualization system are more and more used in the medical field. Indeed such simulators allow the reproduction of various types of operations in order to train, to teach, to check the knowledge and the know-how, and to try new techniques to validate them. These simulations offer several advantages (training without any risks for the patients, economy of time and money, greater availability of the operating room...). Thus, in order to allow the medical team a greater interactivity with the simulator, a visual interface was developed using the MotionDesk software [15]. The user can watch the scene in real-time on a screen and can observe the location of the instrument in comparison with the arm including various points of view at the same time (Fig. 7). To make this possible, it was necessary to use some CAD models of the mechanical component or to digitize some other such as the arm.

\subsection{Definition of the different frames}

The simulator enables the study of the needle paths during an implant insertion. Figure 8 represents the different frames associated with the measure- 


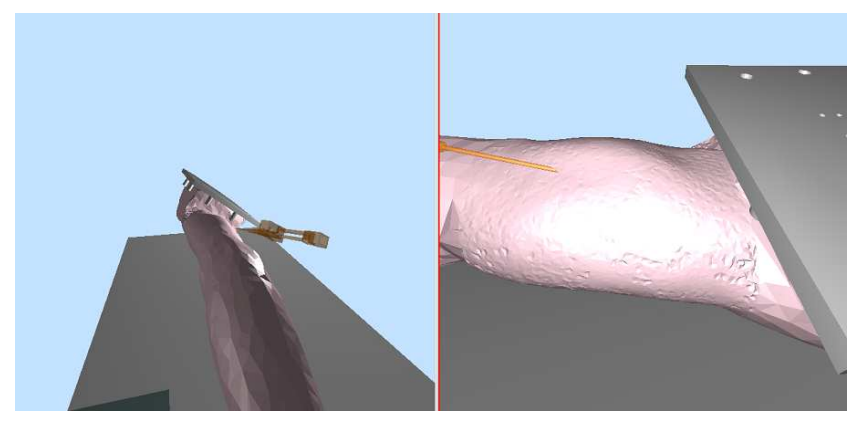

Fig. 7. The visual component

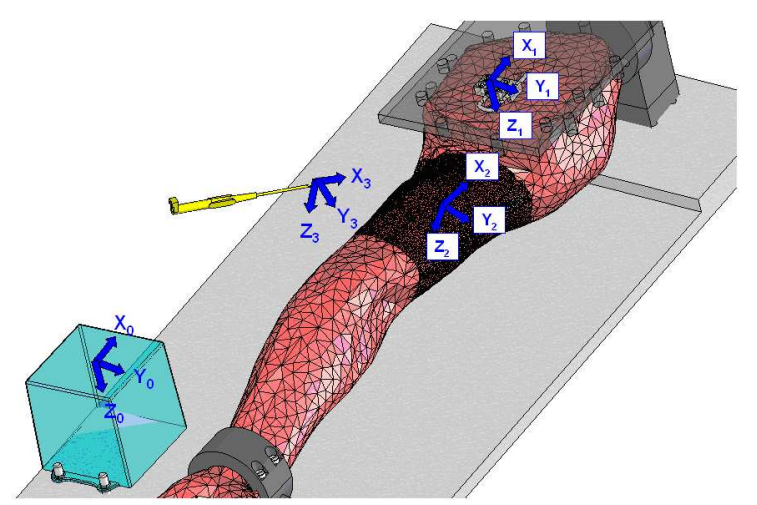

Fig. 8. Definition of the different frames

ment. Frame 0 corresponds to the world frame of the simulator (center of the transmitter), while frame 1 is attached to the sensor on the shoulder. Frame 2 is associated to the insertion task. The $\mathrm{X}, \mathrm{Y}$ and $\mathrm{Z}$ axes of this frame define a tangent plane to the impact point of the needle tip and inside which the path analysis will be carried out. Frame 3' (resp. frame 3") is associated to the sensor of the applicator (resp. obturator). Finally frame 3 is attached to the needle tip. Every frame transformation is based on the homogeneous transformation matrix theory [16]. Given the matrix :

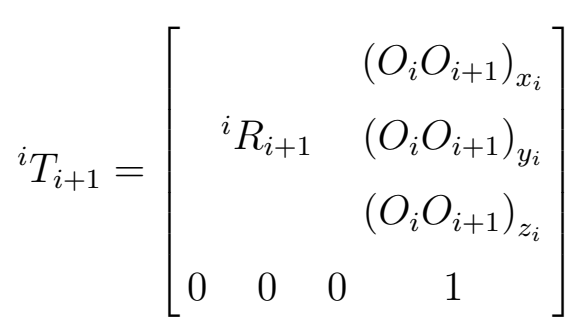

Where:

$-{ }^{i} R_{i+1}$ is the rotation matrix from frame $i$ to frame $i+1$;

- ${ }^{i} T_{i+1}$ is the homogeneous transformation matrix from frame $i$ to frame $i+1$. 
The transformation matrix ${ }^{i} T_{i+1}$ gives the position of $O_{i+1}$ in frame $i$, as defined by $\left(O_{i}, \overrightarrow{x_{i}}, \overrightarrow{y_{i}}, \overrightarrow{z_{i}}\right) .\left(O_{i} O_{i+1}\right)_{x i}\left(\left(O_{i} O_{i+1}\right)_{y i}\right.$ and $\left(O_{i} O_{i+1}\right)_{z i}$, respectively) corresponds to the $x$ ( $y$ and $z$, respectively) coordinate of $\overrightarrow{O_{i} O_{i+1}}$ in frame $i$. Since the most interesting point to follow is the needle tip, we have carried out a frame transformation to establish the coordinates of the needle tip with respect to the frame associated to the task (frame 2).

$$
{ }^{2} T_{3}={ }^{2} T_{1}^{1} T_{0}^{0} T_{3^{\prime}}^{3^{\prime}} T_{3}
$$

The homogeneous transformation matrices directly given by the sensors with respect to the world frame 0 are: ${ }^{0} T_{1}$ and ${ }^{0} T_{3^{\prime}} \cdot{ }^{2} T_{1}$ gives the position and the orientation of the sensor attached to the shoulder in frame 2, as defined by $\left(\mathrm{O}_{2}, \overrightarrow{x_{2}}, \overrightarrow{y_{2}}, \overrightarrow{z_{2}}\right)$. This homogeneous transformation matrix is given by the arm geometry and an accurate fitting of the numerical model with respect to the mechanical one. ${ }^{1} T_{0}$ is the inverse of the homogeneous transformation matrix ${ }^{0} T_{1}$. The matrix ${ }^{3} T_{3}$ gives the position and the orientation of the sensor attached to the applicator in frame 3 . This homogeneous transformation matrix is given by instrument geometry which is accurately known.

\subsection{Criteria of the manipulation evaluation}

In accordance with a physician, three criteria have been selected in order to proceed to manipulation evaluation. These include:

- the arm orientation;

- the location of the impact point;

- the maximum depth reached by the instrument at the end of the manipulation.

A good orientation of the arm is indeed a necessary condition for the success of the insertion because it has a direct effect on the final placement of the implant: the arm has to be positioned in supination in order to make the access to the insertion site easier and only three of the five proposed orientations for the arm are correct. Moreover the location of the impact point has a crucial importance. In practice, it must be in a relatively reduced zone just above the elbow. Finally the implant must not be inserted in the muscle because its removal can be difficult if it is impalpable $[17,18]$. This criterion can be controlled by checking the final depth of the instrument tip. The limit is arbitrarily fixed to $4 \mathrm{~mm}$ which corresponds to the thickness of the skin for an average patient. These three check-points allow the evaluation algorithm implemented in the simulator software component to analyze the data stemming from the sensors. The simulator checks if the arm is in one of the three correct orientations, detects the location of the impact point, records the impact point coordinates, 
checks if the impact point is in the target zone and finally verifies if the depth of the instrument tip passes over a threshold characterizing the border of the aponeurosis. To compute these criteria several problems had to be overcome.

\subsection{Detection of the impact point}

A large part of our work has been the fitting of the numerical model with respect to the mechanical one. Indeed it is necessary that the virtual animation corresponds to reality as accurately as possible. Thus, if the operator moves the insertion instrument, his manipulation must be exactly reproduced on the graphical interface. After the digitization stage of the components (i.e. the support, arm, applicator and obturator), it has been important to fit the different numerical models with the physical model. Moreover, in order to analyze the different needle tip paths, a rectangular skin area of $5 \mathrm{~cm}$ by $3.5 \mathrm{~cm}$ (interest zone) was meshed and stored in a 2D look-up table. The actual location of the instrument tip is compared in real-time with this reference table to detect the impact point by interpolation. At this point it was possible to attach a task frame inside of which the path analysis was carried out. This frame determines the tangent plane of any impact point belonging to the interest zone.

The method used to solve this problem consists in meshing a small zone around the impact point and approximating it with a polynomial function. Figure 9 shows the characteristic of the interest zone. This shape indicates a complex behavior of the skin around the impact point. This is why we have considered a polynomial function of the following form:

$$
z=f(x, y)=\sum_{i=0}^{n} \sum_{j=0}^{m} a_{i j} x^{i} y^{j}=a_{00}+\ldots+a_{n m} x^{n} y^{m}
$$

Where $x, y$ and $z$ are the cartesian coordinates of the needle tip in the frame 2 (Fig. 9).

A criterion based on the norm of the mean error is used to select the order of each polynomial function fitting the data. Several approximations have been made to find the best trade-off between the complexity and the accuracy of the model. The error fitted in Fig. 10 describes the results obtained with a fifth order polynomial approximation $(\mathrm{n}=5 ; \mathrm{m}=5)$. The maximal error is lesser than $0.3 \mathrm{~mm}$ which is consistent with the accuracy predicted for the task.

This approximation is finally used to determine analytically the tangent plane 


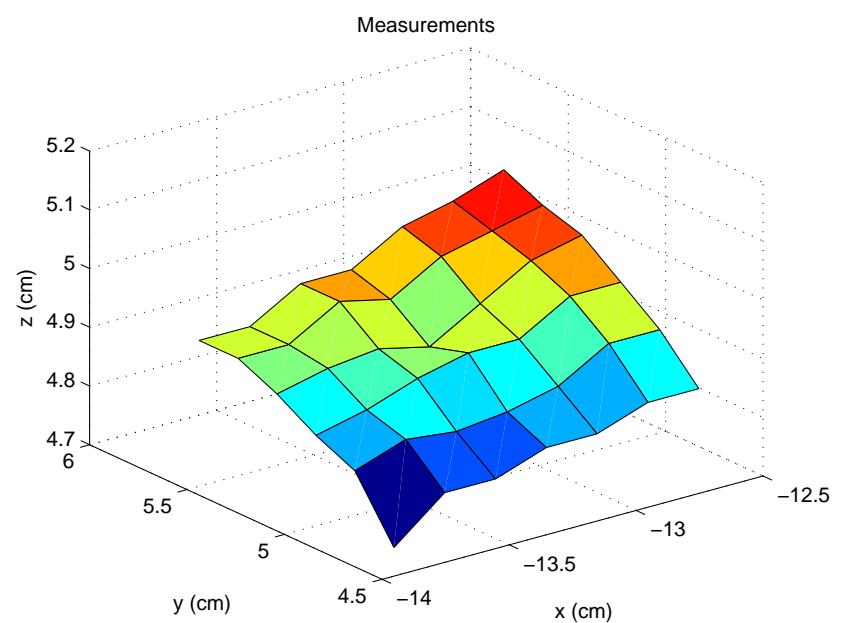

Fig. 9. Characteristic of the skin

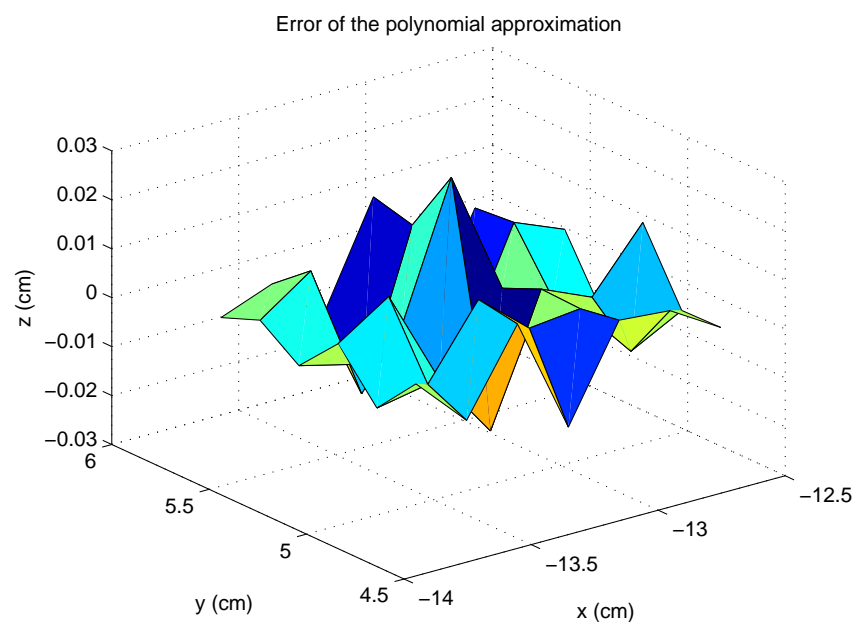

Fig. 10. Approximation error

equation at the impact point $M_{0}\left(x_{0}, y_{0}, z_{0}\right)$ (Fig. 11).

$$
z=z_{0}+\left.\frac{\partial f(x, y)}{\partial x}\right|_{M_{0}}\left(x-x_{0}\right)+\left.\frac{\partial f(x, y)}{\partial y}\right|_{M_{0}}\left(y-y_{0}\right)
$$

The evaluation of the quantitative criteria exposed in section 3.4 has been carried out from the task frame attached to this plane. More particularly, the tangent plane is used to evaluate the depth reached by the needle tip from the skin. 


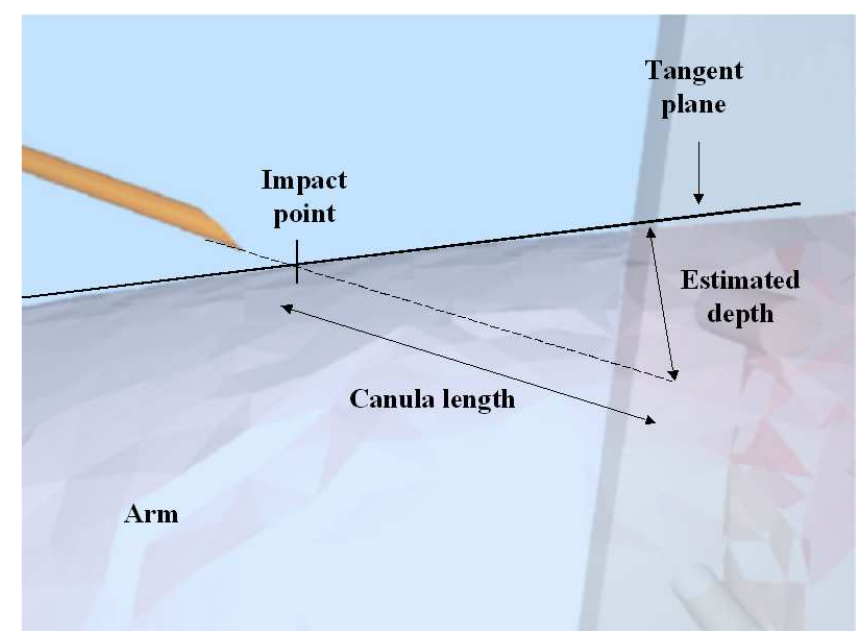

Fig. 11. The tangent plane

\section{Experimental results}

\subsection{Experimental protocol}

A mask was put on the interest zone of the upper arm. We selected seven junior operators and one experienced senior operator. At first the instructor asked each operator to place the arm in the correct orientation. Then the examiner let the candidate palpate the arm and point out the location where he intended to insert the implant. The mask was removed and the candidate inserted the instrument into the impact point. The instructor gave the signal to start the maneuver and simultaneously started recording the trajectory. The operator performed the maneuver and the instructor terminated the recording when the operator considered the maneuver complete.

\subsection{Results and discussion}

Figure 12 illustrates the different recorded paths. Each path corresponds to the needle tip path. Axis X stands for progress along the length of the arm and axis $\mathrm{Z}$ for the insertion path of the instrument in the skin. The zero altitude on axis $\mathrm{Z}$ corresponds to the skin altitude at the impact point whereas the value of $-4 \mathrm{~mm}$ represents the upper bound of the aponeurosis (depth advised for the implant placement). After an a posteriori treatment of data, the paths have been analyzed according to the different evaluation criteria presented in section 3.4. These results are summarized in Tab. 1. For each operator, the 
first (resp. the second) column gives the correctness of the arm position (resp. of the impact point). The third column gives the depth reached by the needle tip at the end of the manipulation projected in the $\vec{x} O \vec{z}$ plane (Fig. 12).

Table 1

manipulation EVALUATION

\begin{tabular}{|c|c|c|c|}
\hline \hline Operator & $\begin{array}{c}\text { Is the arm } \\
\text { orienta- } \\
\text { tion } \\
\text { good? }\end{array}$ & $\begin{array}{c}\text { Is the } \\
\text { impact } \\
\text { point in } \\
\text { the } \\
\text { interest } \\
\text { zone }\end{array}$ & $\begin{array}{c}\text { Depth } \\
\text { reached } \\
\text { by the } \\
\text { needle tip } \\
\text { (mm) }\end{array}$ \\
Novice 1 & Yes & Yes & -0.8 \\
Novice 2 & Yes & Yes & -1.4 \\
Novice 3 & Yes & Yes & -3.4 \\
Novice 4 & Yes & Yes & 0.0 \\
Novice 5 & Yes & Yes & -3.7 \\
Novice 6 & Yes & Yes & -8.3 \\
Novice 7 & Yes & Yes & -1.3 \\
Expert & Yes & Yes & -2.0 \\
\hline \hline
\end{tabular}

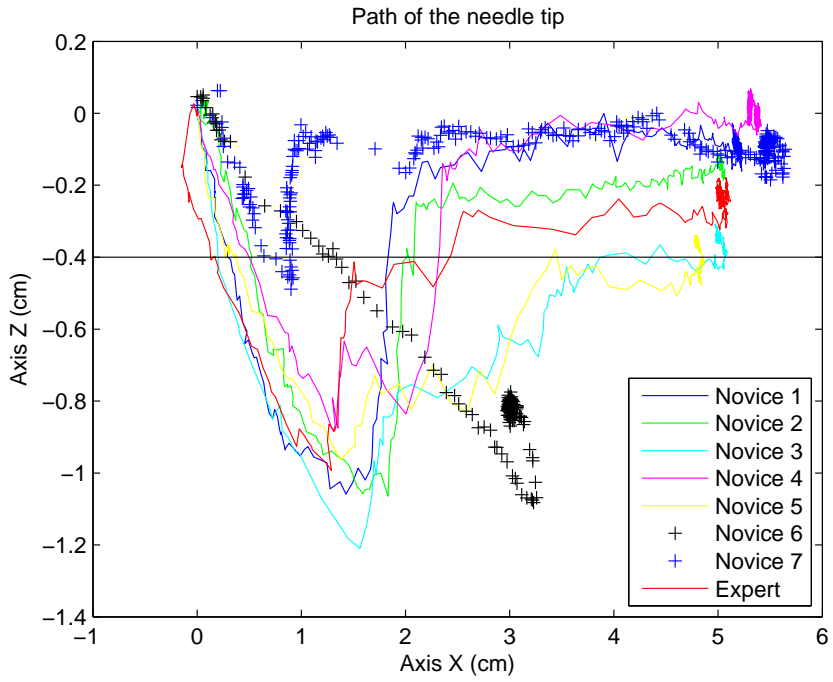

Fig. 12. Paths of the needle tip for the several operators 
Concerning the first experimental results obtained with our simulator,we can observe that all operators have succeeded in the first two control points which are the correctness of the arm orientation and of the impact point localization (Tab. 1). The depth analysis shows that, except the sixth operator, all the operators have managed to place the implant at a correct depth (final depth inferior to $4 \mathrm{~mm}$ ). The final depth of the needle for the third and fifth operators is close to the critical value. One can also remark that, during the manipulation, almost all of the operators went far below the accepted depth : this corresponds actually to the fact that the operator has to go deeper than $4 \mathrm{~mm}$ in order to pierce the skin. But this is not really a criterion that allows concluding a bad insertion. Indeed, the correct placement of the implant is only related to the final depth reached at the end of the manipulation. In fact the theoretical knowledge required to use the insertion instrument is not well documented and no manual currently describes the path of the needle in space. Subcutaneous implant insertion involves a maneuver requiring the operator to represent in his head the three-dimensional positions of the needle tip and the inside of the arm (Fig. 12). This maneuver is carried out blindly and takes place in the arm, making it difficult for an inexperienced doctor to check that it is carried out correctly. Furthermore this kind of maneuver is not frequently performed. The system used in this paper makes it possible to carry out training in a context free of these constraints and provides the first mean of visualizing a maneuver that, until now, has been performed blindly. The simulator and the instrumented tool that we have designed make it possible to improve theoretical knowledge concerning the use of this instrument and to teach the procedure. The implant insertion procedure should be repeated and analyzed, with training adapted to the operator as a function of the results obtained. Finally this system can be used for operator evaluation, comparing junior and senior doctors' trajectories. Recent studies have demonstrated the value of simulation in obstetrics [19-23], in laparoscopic surgery [24,25], in endoscopic surgery [26,27], in orthopedic surgery [28] or in otologic surgery [29]. Studies are currently underway to evaluate the value of these new teaching techniques [30].

\section{Conclusion}

This paper presents a new medical simulator for subcutaneous contraceptive implant insertion. The goal of such a project is to propose a tool that will be used for the improvement of implant insertion conditions and the reduction of the number of difficult removal cases. This simulator can be seen as a good training tool for novices and as a potential device for certifying physicians to insert implants on real patients. The obvious benefit of this simulator is that it will increase patients' security. Considering the physician's manipulation 
constraints, a mechanical simulator has been designed. Composed of a material and a software component, the simulator offers both haptic and graphical interfaces. Novices are able to train themselves progressively with the help of a visual training system and to perform their manipulation a number of times. Several qualitative and quantitative check-points have been set in collaboration with physicians so as to evaluate the correctness of the manipulation. A correct manipulation is judged by the orientation of the arm, the impact point and the depth reached by the instrument at the end of the manipulation. At present a prototype has been manufactured which works under two modes: one for the training and another for the certification. This has allowed us to carry out first experiments. After analyzing several candidates' paths and comparing this data with reference data obtained with an expert, we have validated the principles of the simulator. Nevertheless a more comprehensive study of a novices' larger population should be carried out.

\section{Acknowledgment}

The authors are deeply grateful to Christophe Ducat and Richard Moreau for their technical help and participation in this project.

\section{References}

[1] L. Bensouda-Grimaldi, A. Jonville-Bera, F. Beau-Salinas, S. Llabres, E. AutretLeca, Insertion problems, removal problems, and contraception failures with Implanon ®, Gynécologie obstétrique et fertilité 33 (2005) 986-990.

[2] E. Linn, Progress in contraception: new technology, International Journal of Fertility and Women's Medicine 48 (4) (2003) 182-191.

[3] V. Brache, A. Faundes, F. Alvarez, Risk-benefit effects of implantable contraceptives in women, Expert Opinion on Drug Safety 2 (3) (2003) 321332 .

[4] H. Croxatto, J. Urbancsek, R. Massai, H. C. Bennink, A. van Beek, A multicentre efficacy and safety study of the single contraceptive implant implanon. implanon study group, Human Reproduction 14 (4) (1999) 976-981.

[5] S. Funk, M. Miller, D. Mishell, D. Archer, A. Poindexter, J. Schmidt, E. Zampaglione, Safety and efficacy of Implanon, a single-rod implantable contraceptive containing etonosgestrel, Contraception 71 (2005) 319-326.

[6] L. Mascarenhas, Insertion and removal of implanon: practical considerations, European Journal of Contraception \& Reproductive Health Care 5 Suppl 2 (2000) 29-34. 
[7] G. Wechselberger, D. Wolfram, P. Pülzl, E. Soelder, T. Schoeller, Nerve injury caused by removal of an implantable hormonal contraceptive, American Journal of Obstetrics and Gynecology (AJOG) 195 (1) (2006) 323-326.

[8] L. Mascarenhas, Insertion and removal of Implanon ®), ISSN 0010-7824, 1998.

[9] I. S. Fraser, The challenges of location and removal of implanon contraceptive implants., J Fam Plann Reprod Health Care 32 (3) (2006) 151-152.

[10] P. Faucher, Implanon: insertion problems, removal problems, and contraception failures, Gynécologie obstétrique et fertilité 34 (3) (2006) 283-284.

[11] http://www.enasco.com/.

[12] Ascension Technology, miniBIRD: Installation and operation guide, Standalone and Multiple Transmitter/Multiple Sensor Configurations (May 2001).

[13] D-Space, ControlDesk Installation and Operation Guide (2007).

[14] http://www.dspace.fr.

[15] D-Space, MotionDesk Installation and Operation Guide (2007).

[16] J. Davidson, K. Hunt, Homogeneous transformation matrix theory, Oxford University Press, 2004.

[17] M. Singh, D. Mansour, D. Richardson, Location and removal of non-palpable implanon implants with the aid of ultrasound guidance, Journal of Family Planning and Reproductive Health Care 32 (3) (2006) 153-156.

[18] G. Merki-Feld, C. Brekenfeld, B. Migge, P. Keller, Nonpalpable ultrasonographically not detectable implanon rods can be localized by magnetic resonance imaging, Contraception 63 (6) (2001) 325-328.

[19] R. Silveira, M. Pham, T. Redarce, M. Bétemps, O. Dupuis, A new mechanical birth simulator : BirthSIM, in: IEEE/RSJ International Conference on Intelligent Robots and Systems (IROS'04), Sendai, Japan, 2004, pp. 3948-3954.

[20] O. Dupuis, R. Silveira, A. Zentner, A. Dittmar, P. Gaucherand, M. Cucherat, T. Redarce, R.C.Rudigoz, Birth simulator: Reliability of transvaginal assessment of fetal head station as defined by the American College Obstetricians and Gynecologists classification, American Journal of Obstetrics and Gynecology (AJOG) 192 (2005) 868-874.

[21] R. Moreau, M. Pham, R. Silveira, T. Redarce, X. Brun, O. Dupuis, Design of a new instrumented forceps: application to a safe obstetrical forceps blade placement, IEEE Transactions on Biomedical Engineering 54 (7) (2007) 12801290.

[22] R. H. Allen, B. R. Bankoski, D. A. Nagey, Simulating birth to investigate clinician-applied loads on newborns, Medical Engineering \& Physics 17 (5) (1995) 380-384. 
[23] R. Allen, S. Cha, L. Kranker, T. Johnson, E. Gurewitsch, Comparing mechanical fetal response during descent, crowning, and restitution among deliveries with and without shoulder dystocia, American Journal of Obstetrics and Gynecology (AJOG) 196 (6).

[24] R. Baumann, W. Maeder, G. Glauser, The pantoscope: A spherical remotecenter-of-motion parallel manipulator for force reflection, in: IEEE International Conference on Robotics and Automation (ICRA'97), Albuquerque, New Mexico, USA, 1997, pp. 743-750.

[25] S. Undre, A. Darzi, Laparoscopy simulators, Journal of Endourology 21 (3) (2007) 274-279.

[26] The KIZMET simulation homepage, http://iregt1.iai.fzk.de/, website checked on 20-04-2006.

[27] M. Karouia, P. Arhets, Y. Aigrain, A novel design of endoscopic surgery training simulator, in: International Symposium on Robotics (ISR'04), Paris, France, 2004, p. 66.

[28] P. Zambelli, C. Bregand, S. Dewarrat, Planning and navigation solution in resurfacing hip surgery: a way to reduce the surgical approach, in: Poster session, $3^{\text {rd }}$ Annual Meeting of the International Society for Computer Assisted Orthopaedic Surgery, Marbella, Spain, 2003.

[29] R. Grunert, G. Strauss., H. Moeckel, M. Hofer, A. Poessneck, U. Fickweiler, M. Thalheim, R. Schmiedel, P. Jannin, T. Schulz, J. Oeken, A. Dietz, W. Korb, Elephant - an anatomical electronic phantom as simulation-system for otologic surgery, in: IEEE International Conference of the Engineering in Medicine and Biology Society (IEEE EMBC'06), New York, USA, 2006, pp. 4408-4411.

[30] N. Oshima, M. Aizudding, R. Midorikawa, J. Solis, Y. Ogura, A. Takanishi, Development of a suture/ligature training system designed to provide quantitative information of the learning progress of trainees, in: IEEE International Conference on Robotics and Automation (ICRA'07), Roma, Italy, 2007, pp. 2285-2291. 